\title{
Association between Daily Living Walking Speed and Lifestyle and Physiological Factors in Older, Female Pokémon GO Players
}

\author{
Shuhei Nomura1,2*, Akifumi Eguchi1,3*, Daisuke Yoneoka1,4*, Takayuki Kawashima1,5*, \\ Norimichi Hirahara', Yuta Tanoue1,6, Stuart Gilmour ${ }^{4}$, Hisateru Tachimori7,8, Hiroaki Miyata1" \\ ${ }^{1}$ Department of Health Policy and Management, School of Medicine, Keio University, Tokyo, Japan \\ ${ }^{2}$ Department of Global Health Policy, Graduate School of Medicine, The University of Tokyo, Tokyo, Japan \\ ${ }^{3}$ Department of Sustainable Health Science, Center for Preventive Medical Sciences, Chiba University, Chiba, Japan \\ ${ }^{4}$ Graduate School of Public Health, St. Luke’s International University, Tokyo, Japan \\ ${ }^{5}$ Department of Mathematical and Computing Science, Tokyo Institute of Technology, Tokyo, Japan \\ ${ }^{6}$ Institute for Business and Finance, Waseda University, Tokyo, Japan \\ ${ }^{7}$ Department of Clinical Epidemiology, Translational Medical Center, National Center of Neurology and Psychiatry, Tokyo, Japan \\ ${ }^{8}$ Endowed Course for Health System Innovation, School of Medicine, Keio University, Tokyo, Japan \\ Email: ${ }^{*} h-m @ k e i o . j p$
}

How to cite this paper: Nomura, S., Eguchi, A., Yoneoka, D., Kawashima, T., Hirahara, N., Tanoue, Y., Gilmour, S., Tachimori, H. and Miyata, H. (2021) Association between Daily Living Walking Speed and Lifestyle and Physiological Factors in Older, Female Pokémon GO Players. Health, 13, 564-573.

https://doi.org/10.4236/health.2021.135042

Received: April 13, 2021

Accepted: May 18, 2021

Published: May 21, 2021

Copyright $\odot 2021$ by author(s) and Scientific Research Publishing Inc. This work is licensed under the Creative Commons Attribution International License (CC BY 4.0).

http://creativecommons.org/licenses/by/4.0/ (c) (i) Open Access

\begin{abstract}
Background: Walking speed is a reliable barometer of adverse health outcomes, particularly among older people. Few studies evaluated factors associated with daily actual walking speed, rather than that measured in a laboratory setting or self-reported data. Methods: In a joint effort with The Pokémon Company, we recruited study participants through a women's magazine and analyzed data from 63 Pokémon GO players. We measured the true walking speed in daily life among older women using a data-gathering mobile application. Then, using questionnaire survey data collected on these participants in 2019, we estimated the relationship between walking speed and their lifestyle and physiological factors. In the analysis, we employed a bi-directional stepwise linear regression approach, with the Akaike information criterion (AIC) for variable selection. Results: The mean age of the 63 participants was 63.03 years (standard deviation, SD 7.46); the average walking speed was 4.56 $\mathrm{km}$ per hour (SD 1.54); and $52(82.5 \%)$ and 55 (87.3\%) participants answered that they had excellent/good health physically and mentally, respectively. After adjusted for covariates in a stepwise regression approach, we identified a significant association between -1.33 (95\% confidence intervals -2.52 to $-0.15) \mathrm{km} /$ hour lower walking speed and the experience of outdoor falls within a year. We also demonstrated that the walking speed was 1.07 (0.33 to
\end{abstract}


1.81) $\mathrm{km} /$ hour faster for those who had played Pokémon GO before the study started. Conclusions: The true walking speed in daily life among older women was measured by a data-gathering mobile application. Although all participants were women recruited on a voluntary basis with an understanding of the purpose of the study, and the surveys were conducted at a cross-sectional setting, a significant relationship with the experience of outdoor falls was demonstrated. We also present evidence suggesting a possible relationship between Pokémon GO play and faster walking speed.

\section{Keywords}

Daily Actual Walking Speed, Pokémon GO Players, Experience of Outdoor Falls

\section{Introduction}

Because walking is a complex functional activity, many factors contribute to or affect walking speed, such as individual health status, sensory and perceptual functions, muscle performance and musculoskeletal condition, motor function, habitual activity levels, cognitive status, and mental health [1]. Walking speed is thus an important measure for evaluating physical function, especially in older people, because their lower-leg muscular strength, balance, and other functional capacities may be impaired. There is also some evidence suggesting that walking speed is a powerful measure of health status, such as that it could be a prognostic factor for vascular events in stroke patients [2] and a survival predictor in cancer patients [3]. In recent years, the importance of prevention and amelioration of frailty-a clinical state with increased age-related vulnerability to dependence, long-term residential care, cognitive decline, institutionalization, and even mortality after a stressor event-has been increasing. Various studies have found that walking speed is a useful diagnostic indicator of frailty [4]. Walking speed is, therefore, a reliable barometer of adverse health outcomes particularly among older people.

Although many approaches have been used to improve walking and positive results of interventions have been reported [5], studies considering multiple factors related to walking performance are lacking. In addition, many studies evaluating walking speed are based on self-reported data or use data measured on an artificial walkway in laboratory settings where people may intentionally alter their walking speed. Walking speed measured in the laboratory is known to be different from the true walking speed in daily life [6].

On the other hand, it has recently become possible to evaluate walking speed in daily life with various devices, such as smartphones [7]. In this study, we estimated the relationship between the true walking speed in daily life in older women and their lifestyle and physiological factors on daily life activities and quality of life, using mobility data measured by a data-gathering mobile application called "Journeys" and questionnaire data collected from the two surveys. 


\section{Methods}

\subsection{Settings}

This is a prospective cohort study designed by us with the cooperation of The Pokémon Company and Realize Mobile Communications Corp. (a commercial partnership of Sentiance in Japan's markets) for the use of mobility data collected by "Journeys" among female Pokémon GO players. Journeys is a globally recognized and used app developed by Sentiance that automatically creates users' personal behavioral profile based on their mobility patterns throughout the day, by analyzing their mobile phone's sensor data [8]. Taking into account any combination of available data (accelerometer, gyroscope, motion activities, location), the machine learning models developed by Sentiance detect walking, running, biking, driving, and other transport modes. Details can be found elsewhere [9]. This application tracks and records users' mobility even when it is closed, if users configure the application to "always" enable location permission. These data are automatically acquired and stored by Sentiance.

The study participants were recruited through the advertising page of a monthly subscription magazine about health and living for senior women issued by Halmek Corp. on August 10, 2019. Applications to participate in the study were accepted from September 30, 2019 to March 31, 2020, and a 500 JPY (approximately 5 USD) gift card was provided to each participant. There were no inclusion or exclusion criteria for participants. The analysis of the participants' mobility data started after they completed two questionnaire surveys that we conducted, with voluntary participation. Each participant's mobility data stored in Sentiance since the second survey was aggregated by Sentiance into weekly data on walking duration and distance, and was provided to us. The determination of the sample size was not able to be strictly considered due to the nature of participant recruitment method.

The survey response data and mobility data were linked on an individual level, by referring to the Journeys user identification provided by the survey participants. In addition to whether the respondents were Pokémon GO players or not, the first survey asked about their subjective health. The respondents scanned a QR code in the magazine, jumped to the online survey page, and submitted their answers to the questionnaire. The respondents of the first survey were invited by email to an in-person research briefing held by The Pokémon Company and completed the second survey asking about lifestyle and physiological factors. Those who had not installed Pokémon GO and Journeys on their smartphone at the time, were instructed to install them there. The research briefings were held eight times from September 30, 2019 to March 31, 2020 (once in Osaka and seven times in Tokyo), so the start of the analysis period varied for each participant. There were 801 participants in the first survey and 97 participants in the second survey. Data from 66 participants who have installed Journeys and agreed to participate in this study were used in the analyses. Questions in the surveys are outlined in the resulting table. 


\subsection{Data Analysis}

Since the observation period varies among participants, the weekly average walking speed, which was calculated from the weekly walking duration and distance during the period, was further averaged over the observed weeks. Unless otherwise indicated, it was treated as the average walking speed of each participant in the study. Then, we performed bi-directional stepwise multivariable linear regression to identify the factors associated with the walking speed, where the Akaike information criterion (AIC) was used for stepwise variable selection. Responses to multiple-choice questions were converted to binary data prior to analysis. Ordinal variables were included successively as discrete variables.

\subsection{Ethical Approval}

Ethical approval for this study was granted by the Ethics Committee of the Graduate School of Health Management, Keio University; approval number 2019-03. Written informed consent was obtained for each participant.

\section{Results}

Out of 66 female participants, we excluded three participants who had missing data on the survey or had no walking duration and distance data, possibly because the permission to use location information was disabled. None of the 63 participants were lost to follow-up by the end of the study period. The mean age of the 63 participants was 63.03 years (standard deviation, SD 7.46) (Table 1). The mean of the average walking speed of the participants was $4.56 \mathrm{~km}$ per hour (SD 1.54). Twenty-eight (44.4\%) participants were Pokémon GO players before the study started. Thirty-three (52.4\%) participants reported drinking habits and one (1.6\%) reported daily smoking habits. A total of $30(47.6 \%)$ participants indicated that they did not have any diseases under treatment, and $52(82.5 \%)$ and 55 (87.3\%) participants answered that they had excellent/good health physically and mentally, respectively.

After the stepwise variable selection process from the variables presented in Table 1 using AIC, age, a history of Pokémon GO play before the study, confidence in physical strength, lifestyle habits to stay healthy, experience of outdoor falls within a year, mode of transportation used when going out, and presence of disease under treatment remained in the final regression models (Table 2). We found that, after adjusting for covariates, those who had played Pokémon GO before the study started walked $1.07 \mathrm{~km} /$ hour (95\% confidence interval, CI 0.33 to 1.81) faster than those who did not. The walking speed among those who had experience of outdoor falls within a year was $1.33 \mathrm{~km} /$ hour (95\% CI 0.15 to 2.52 ) slower than those who did not. Those who reported that they use a car when they go out walked $0.81 \mathrm{~km} /$ hour ( $95 \%$ CI 0.15 to 1.47 ) faster than those who did not.

\section{Discussion}

It is important to note that this study is limited to female Pokémon GO players. 
Table 1. Characteristics of the study participants.

\begin{tabular}{|c|c|}
\hline Variables & Number (percent) \\
\hline \multicolumn{2}{|l|}{ 1. Demographic data } \\
\hline Walking speed (mean [SD]) & $4.56(1.54)$ \\
\hline Age (mean $[\mathrm{SD}]$ ) & $63.03(7.46)$ \\
\hline Pokémon GO player before the study started (SC) & $28(44.4)$ \\
\hline \multicolumn{2}{|l|}{ 2. Subjective health view } \\
\hline \multicolumn{2}{|l|}{ Health consciousness (SC) } \\
\hline Ver high & $12(19.0)$ \\
\hline High & $47(74.6)$ \\
\hline Fair & $4(6.3)$ \\
\hline Poor & $0(0.0)$ \\
\hline \multicolumn{2}{|l|}{ Physical health status (SC) } \\
\hline Excellent & $1(1.6)$ \\
\hline Good & $51(81.0)$ \\
\hline Fair & $10(15.9)$ \\
\hline Poor & $1(1.6)$ \\
\hline \multicolumn{2}{|l|}{ Mental health status (SC) } \\
\hline Excellent & $7(11.1)$ \\
\hline Good & $48(76.2)$ \\
\hline Fair & $8(12.7)$ \\
\hline Poor & $0(0.0)$ \\
\hline \multicolumn{2}{|l|}{ Confidence in physical strength (SC) } \\
\hline Very high & $1(1.6)$ \\
\hline High & $29(46.0)$ \\
\hline Medium & $29(46.0)$ \\
\hline Low & $4(6.3)$ \\
\hline \multicolumn{2}{|l|}{ Sleep quality (SC) } \\
\hline Very good & $3(4.8)$ \\
\hline Good & $37(58.7)$ \\
\hline Poor & $22(34.9)$ \\
\hline Very poor & $1(1.6)$ \\
\hline \multicolumn{2}{|l|}{ Hearing quality (SC) } \\
\hline Very good & $8(12.7)$ \\
\hline Good & $33(52.4)$ \\
\hline Fair & $21(33.3)$ \\
\hline Poor & $1(1.6)$ \\
\hline
\end{tabular}




\section{Continued}

Very poor

$0(0.0)$

Vision quality (SC)

Very good

Good

$24(38.1)$

Fair

Poor

3. Lifestyle

Mode of transportation used when going out (MC)

Car

Public transportation

Lifestyle habits to stay healthy (MC)

Healthy eating

Avoid building up stress

$32(50.8)$

Exercise

$36(57.1)$

Talking with family and friends

Volunteer work

$29(46.0)$

$4(6.3)$

Taking a health check-up

$27(42.9)$

Gargle and hand wash

$30(47.6)$

Adequate sleep and rest

$48(76.2)$

Early to bed and early to rise

$15(23.8)$

Drink alcohol (SC)

$33(52.4)$

Daily smoke (SC)

$1(1.6)$

\section{Health conditions}

Use of dental prosthesis (MC)

Removable partial denture

Fixed bridge

$18(28.6)$

Dental implant

None

$34(54.0)$

Experience of indoor falls within a year (SC)

Experience of outdoor falls within a year (SC)

$9(14.3)$

Diseases under treatment (MC)

$\begin{array}{cc}\text { Hypertension } & 15(23.8) \\ \text { Diabetes } & 4(6.3) \\ \text { Dyslipidemia } & 11(17.5) \\ \text { Eye disease } & 8(12.7)\end{array}$

SD: standard deviation; SA: single-choice question; MA: multiple-choice question. ${ }^{\star}$ 
Table 2. Results of the multivariable regression analyses-factors associated with walking speed $(\mathrm{km} /$ hour$)$.

\begin{tabular}{cccc}
\hline & Coefficient & 95\% CI & P-value \\
\hline Age & -0.04 & -0.09 to 0.01 & 0.17 \\
Pokémon GO player before the study started & 1.07 & 0.33 to 1.81 & $<0.01$ \\
Confidence in physical strength & 0.46 & -0.09 to 1.01 & 0.11 \\
Lifestyle habits to stay healthy & & & \\
Taking a health check-up & 0.69 & -0.00 to 1.39 & 0.06 \\
$\begin{array}{c}\text { Experience of outdoor falls within a year } \\
\text { Diseases under treatment }\end{array}$ & -1.33 & -2.52 to -0.15 & $<0.05$ \\
Hypertension & & & 0.07 \\
Eye disease & 0.79 & -0.04 to 1.62 & 0.10 \\
Car & -0.88 & -1.93 to 0.16 & \\
Mode of transportation used when going out & & & 0.18 \\
\hline
\end{tabular}

CI: confidence intervals.

The first survey confirmed that 28 (44.4\%) people had Pokémon GO installed on their smartphones, and during the second survey all participants were prompted to install it. Pokémon GO is a smartphone game application that blends the real and digital worlds. By using GPS (global positioning satellite) location information, it allows players to explore their neighborhoods to find creatures and treasures to use in the game. Therefore, it could be reasonable to think that the study participants were probably biased to a relatively healthier group than the average of their age group, and in fact, most of the participants in this study reported good physical and mental health.

With 1 billion downloads worldwide, Pokémon GO is a virtual health companion thanks to a combination of features that make walking more fun, resulting in increased physical activity and decreased sedentary behaviors with particularly engaged users [10]. Our study demonstrated that the walking speed was 1.07 $\mathrm{km} /$ hour faster for those who have played Pokémon GO before the study started. The frequency of playing Pokémon GO was not a compulsory question, so only 19 of the 28 respondents who had played Pokémon GO before the study began responded, of whom 15 (79\%) said they played every day.

There is evidence that walking speed is significantly associated with fall risk in older adults [11] due to reduced functional strength [1]. In fact, experience of outdoor falls within a year was significantly associated with lower walking speed in the present study. The study finding that people who use a car for going out walk faster is also an indication that higher physical functioning affects walking speed. In addition, a systematic review suggested that walking speed has traditionally been measured in the laboratory on an artificial walkway [12]. In this study, we used smartphone application data to measure walking speed in daily 
life, and our findings were similar to previous studies in terms of risk factor analysis. For example, Huijben et al. and Callisaya et al. measured the walking speed among older people using a computerized walkway system, and highlighted the relationship between lower walking speed and risk of falling [11] [13], as with the present study.

We found no statistically significant associations with some factors, such as self-reported hearing and vision quality, which have been found to be associated with reduced walking speed among older adults [14]. The difference between these previous studies and our study may be attributed largely to the demographics of the study participants. As noted above, the present study participants might appear to be relatively healthy compared to their average same age group. Other limitations of the study were that all participants were women recruited on a voluntary basis with an understanding of the purpose of the study, and because the surveys were conducted in a cross-sectional setting, the direction of any causal relationship could not be established. The small sample size can be attributed to the fact that we relied only on the advertisement pages of the magazine for participant recruitment and the in-person research briefing was held only in two limited locations, Osaka and Tokyo.

Despite these limitations, we believe that this study contributes to the emerging literature on forestalling the decline in physical function among older people globally. Our analyses also indicated that mobility information obtained from a data-gathering mobile application, which is very popular, simple, and accessible to everyone, may be very useful in understanding and capturing the walking speed of older people.

\section{Author Contribution}

Nomura, Eguchi, Yoneoka, Kawashima, Hirahara, Tanoue, and Miyata had full access to all of the data in the study and take responsibility for the integrity of the data and the accuracy of the data analysis. Concept and design: Nomura, Hirahara, Miyata. Acquisition, analysis, or interpretation of data: All authors. Drafting of the manuscript: Nomura. Critical revision of the manuscript for important intellectual content: All authors. Statistical analysis: Eguchi, Yoneoka, Kawashima, Gilmour. Administrative, technical, or material support: Hirahara, Tachimori, Miyata. Supervision: Miyata.

\section{Acknowledgements}

We would like to thank The Pokémon Company and Halmek Corp. for recruiting study participants and conducting the surveys, and Realize Mobile Communications Corp. for introducing the Journeys application for collecting mobility data of the participants.

\section{Funding Statement}

This work was also funded partly by the Japan Science and Technology Agency 
(JST) and The Pokémon Company. The funder of the study had no role in the study design, data analysis, data interpretation, or writing of the paper. The authors had full access to all the data in the study and had final responsibility to submit for publication.

\section{Conflicts of Interest}

Miyata reports grant from the Japan Science and Technology Agency (JST) and The Pokémon Company. Other co-authors declare no competing interests. The study was approved by the appropriate ethics review board.

\section{References}

[1] Fritz, S. and Lusardi, M. (2009) White Paper: "Walking Speed: The Sixth Vital Sign". Journal of Geriatric Physical Therapy, 32, 46-49. https://doi.org/10.1519/00139143-200932020-00002

[2] Kawajiri, H., Mishina, H., Asano, S., Kono, Y., Hayashi, H., Niwa, J.I., Doyu, M., Kimura, S. and Yamada, S. (2019) Maximum Walking Speed at Discharge Could Be a Prognostic Factor for Vascular Events in Patients with Mild Stroke: A Cohort Study. Archives of Physical Medicine and Rehabilitation, 100, 230-238. https://doi.org/10.1016/j.apmr.2018.05.025

[3] Hantel, A., DuMontier, C., Odejide, O.O., Luskin, M.R., Sperling, A.S., Hshieh, T., Chen, R., Soiffer, R., Driver, J.A. and Abel, G.A. (2020) Gait Speed, Survival, and Recommended Treatment Intensity in Older Adults with Blood Cancer Requiring Treatment. Cancer, 127, 875-883. https://doi.org/10.1002/cncr.33344

[4] Fried, L.P., Tangen, C.M., Walston, J., Newman, A.B., Hirsch, C., Gottdiener, J., Seeman, T., Tracy, R., Kop, W.J., Burke, G., McBurnie, M.A. and Cardiovascular Health Study Collaborative Research G. (2001) Frailty in Older Adults: Evidence for a Phenotype. The Journals of Gerontology: Series A, Biological Sciences and Medical Sciences, 56, M146-M156. https://doi.org/10.1093/gerona/56.3.M146

[5] Zhang, W., Low, L.F., Gwynn, J.D. and Clemson, L. (2019) Interventions to Improve Gait in Older Adults with Cognitive Impairment: A Systematic Review. Journal of the American Geriatrics Society, 67, 381-391. https://doi.org/10.1111/jgs.15660

[6] Kawai, H., Obuchi, S., Watanabe, Y., Hirano, H., Fujiwara, Y., Ihara, K., Kim, H., Kobayashi, Y., Mochimaru, M., Tsushima, E. and Nakamura, K. (2020) Association between Daily Living Walking Speed and Walking Speed in Laboratory Settings in Healthy Older Adults. International Journal of Environmental Research and Public Health, 17, 2707. https://doi.org/10.3390/ijerph17082707

[7] Takayanagi, N., Sudo, M., Yamashiro, Y., Lee, S., Kobayashi, Y., Niki, Y. and Shimada, H. (2019) Relationship between Daily and In-Laboratory Gait Speed among Healthy Community-Dwelling Older Adults. Scientific Reports, 9, 3496. https://doi.org/10.1038/s41598-019-39695-0

[8] Sentiance. Journeys App. https://www.sentiance.com/demo/

[9] Sentiance (2016) How AI Drives the Mobile Contextual Revolution. https://www.sentiance.com/2016/05/31/mobile-contextual-revolution/

[10] Nigg, C.R., Mateo, D.J. and An, J. (2017) Pokemon GO May Increase Physical Activity and Decrease Sedentary Behaviors. American Journal of Public Health, 107, 37-38. https://doi.org/10.2105/AJPH.2016.303532 
[11] Callisaya, M.L., Blizzard, L., Schmidt, M.D., Martin, K.L., McGinley, J.L., Sanders, L.M. and Srikanth, V.K. (2011) Gait, Gait Variability and the Risk of Multiple Incident Falls in Older People: A Population-Based Study. Age Ageing, 40, 481-487. https://doi.org/10.1093/ageing/afr055

[12] Binotto, M.A., Lenardt, M.H. and Rodriguez-Martinez, M.D.C. (2018) Physical Frailty and Gait Speed in Community Elderly: A Systematic Review. Revista da Escola de Enfermagem da USP, 52, e03392.

https://doi.org/10.1590/s1980-220x2017028703392

[13] Huijben, B., van Schooten, K.S., van Dieen, J.H. and Pijnappels, M. (2018) The Effect of Walking Speed on Quality of Gait in Older Adults. Gait Posture, 65, 112-116. https://doi.org/10.1016/j.gaitpost.2018.07.004

[14] Li, L., Simonsick, E.M., Ferrucci, L. and Lin, F.R. (2013) Hearing Loss and Gait Speed among Older Adults in the United States. Gait Posture, 38, 25-29.

https://doi.org/10.1016/j.gaitpost.2012.10.006 